University of New Hampshire

University of New Hampshire Scholars' Repository

Space Science Center

Institute for the Study of Earth, Oceans, and

Space (EOS)

1998

\title{
MeV measurements of gamma-ray bursts by CGRO-COMPTEL
}

\author{
A Connors \\ University of New Hampshire - Main Campus \\ W Collmar \\ Max-Planck-Institut für extraterrestriche Physik \\ W Hermsen \\ Space Research Organization of the Netherlands (SRON) \\ S C. Kappadath \\ Louisiana State University - Baton Rouge \\ R M. Kippen \\ University of Alabama - Huntsville
}

See next page for additional authors

Follow this and additional works at: https://scholars.unh.edu/ssc

Part of the Astrophysics and Astronomy Commons

\section{Recommended Citation \\ MeV measurements of gamma-ray bursts by CGRO-COMPTEL Connors, A. and Collmar, W. and Hermsen, W. and Kappadath, S. and Kippen, R. M. and Kuiper, L. and McConnell, M. and Pelaez, F. and Ryan, J. M. and Schönfelder, V. and Varendorff, M. and Williams, O. R. and Winkler, C. and Young, C. A., AIP Conference Proceedings, 428, 344-348 (1998), DOI:http://dx.doi.org/10.1063/1.55465}

This Conference Proceeding is brought to you for free and open access by the Institute for the Study of Earth, Oceans, and Space (EOS) at University of New Hampshire Scholars' Repository. It has been accepted for inclusion in Space Science Center by an authorized administrator of University of New Hampshire Scholars' Repository. For more information, please contact Scholarly.Communication@unh.edu. 


\section{Authors}

A Connors, W Collmar, W Hermsen, S C. Kappadath, R M. Kippen, L Kuiper, Mark L. McConnell, F Pelaez, James M. Ryan, V Schonfelder, M Varendorff, O R. Williams, C Winkler, and C A. Young 


\section{AIP $\mid$ proceedings}

\section{MeV measurements of gamma-ray bursts by CGRO-COMPTEL}

A. Connors, W. Collmar, W. Hermsen, S. Kappadath, R. M. Kippen, L. Kuiper, M. McConnell, F. Pelaez, J. M. Ryan, V. Schönfelder, M. Varendorff, O. R. Williams, C. Winkler, and C. A. Young

Citation: AIP Conference Proceedings 428, 344 (1998); doi: 10.1063/1.55465 View online: http://dx.doi.org/10.1063/1.55465

View Table of Contents:

http://scitation.aip.org/content/aip/proceeding/aipcp/428?ver=pdfcov

Published by the AIP Publishing

\section{Articles you may be interested in}

MeV measurements of $\mathrm{y}$-ray bursts by CGRO-COMPTEL: Revised catalog AIP Conf. Proc. 526, 28 (2000); 10.1063/1.1361501

Simulated observations of gamma-ray bursts with GLAST AIP Conf. Proc. 428, 884 (1998); 10.1063/1.55414

EGRET observations of bursts at MeV energies AIP Conf. Proc. 428, 309 (1998); 10.1063/1.55340

Preliminary spatial analysis of combined BATSE/Ulysses gamma-ray burst locations AIP Conf. Proc. 428, 114 (1998); 10.1063/1.55305

The total cosmic diffuse gamma-ray spectrum from 9 to $30 \mathrm{MeV}$ measured with COMPTEL AIP Conf. Proc. 410, 1218 (1997); 10.1063/1.54105 


\title{
MeV Measurements of Gamma-Ray Bursts by CGRO-COMPTEL
}

\author{
A. Connors ${ }^{1}$, W. Collmar ${ }^{3}$, W. Hermsen ${ }^{4}$, S. Kappadath ${ }^{1}$, \\ R. M. Kippen ${ }^{5}$, L. Kuiper ${ }^{4}$, M. McConnell ${ }^{1}$, F. Pelaez ${ }^{2,6}$, \\ J. M. Ryann ${ }^{1}$, V. Schönfelder ${ }^{3}$, M. Varendorff', O. R. Williams ${ }^{2}$, \\ C. Winkler ${ }^{2}$, and C. A. Young ${ }^{1}$ \\ ${ }^{1}$ Space Science Center, University of New Hamphsire, Durham NH, 09824 USA \\ ${ }^{2}$ Astrophysics Division, ESTEC, NL-2200 AG Noordwijk, NL \\ ${ }^{3}$ Max-Planck-Institut fur Extraterrestrische Physik, D-85740 Garching, FRG \\ ${ }^{4}$ SRON-Utrecht, Sorbonnelaan 2, NL-3584 Utrecht, NL \\ ${ }^{5}$ C.S.P.A.R., University of Alabama in Huntsville, Huntsville AL, 95899 USA \\ ${ }^{6}$ now at USRA, NASA/GSFC, Greenbelt, MD, USA
}

\begin{abstract}
Since the launch of the Compton Gamma-Ray Observatory in April 1991, the imaging COMPTEL telescope has accumulated positions and $0.75-30 \mathrm{MeV}$ spectra of more than thirty gamma-ray bursts within its $\sim \pi$ sr field of view. In an ongoing collaboration with BACODINE/GCN, COMPTEL positions are relayed to a global network of multiwavelength observers in near real time ( 10 minutes). Here we summarize the $\mathrm{MeV}$ properties, and present spatial, spectral, and temporal data for the latest of these events, GRB 970807. In concurrence with earlier SMM and current BATSE, OSSE, and EGRET measurements, COMPTEL data add to the accumulating evidence that GRB spectra do seem to have a characteristic shape: a peak (in $E^{2} F(E)$ ) around several hundred $\mathrm{keV}$; and a power law above (spectral index 1.5-3.5) extending beyond the COMPTEL energy range.
\end{abstract}

\section{INTRODUCTION}

The COMPTEL experiment on board the Compton Gamma-Ray Observatory (CGRO) has accumulated a sample of 33 gamma-ray bursts in its $\sim \pi$ sr field of view since its launch in April 1991. COMPTEL images these events in telescope or double-scatter mode $(0.75-30 \mathrm{MeV})$; and measures burst spectra and light-curves both in telescope mode and in its burst, or spectroscopic single detector mode (0.1$11 \mathrm{MeV}$ ) [1]. These are preferentially bursts with bright $\mathrm{MeV}$ emission. Here we describe some global properties of this sample, and report the details of the latest burst. 


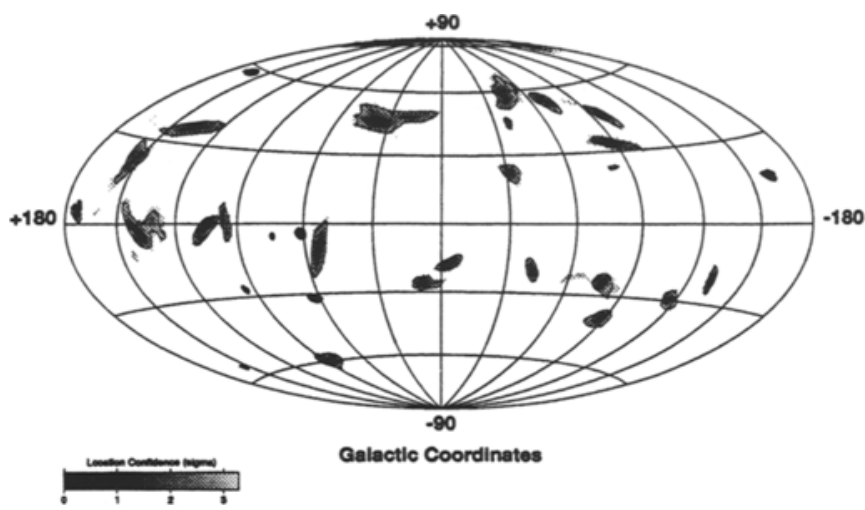

FIGURE 1. All-sky map of COMPTEL positions, in Galactic coordinates.

\section{LOCATIONS}

In Figure 1 we display an all-sky map of all COMPTEL bursts to date. COMPTEL relies on the BATSE trigger for preliminary start-time and position. We then localize each event that falls within $\sim 65^{\circ}$ of telescope zenith, using a maximumlikelihood method [2], with on average $\sim 1.13^{\circ}$ statistical and $\sim 0.5^{\circ}$ systematic accuracy. The grayscale indicates confidence level, with the contours outlining the $3 \sigma$ statistical limits, so the brightest bursts are actually the smallest. A detailed study of bursts during the first four years has so far confirmed that this map is consistent with isotropy [2].

\section{SPECTRA}

In Figure 2 we display a histogram of the photon power-law indices from fits to the $0.75-30 \mathrm{MeV}$ telescope-mode spectra in the COMPTEL sample. Data from the first four years have shown that $0.75-30 \mathrm{MeV}$ spectra tend to be best represented by a power-law, rather than by a form with more curvature $[3,4]$. Since then, we have added spectra from three more bursts. These are also consistent with powerlaw models, both in the $0.75-30 \mathrm{MeV}$ telescope data and the $\sim 0.3-2 \mathrm{MeV}$ burst mode data (GRBs 960808 and 961212: [5]; GRB 970807, this work). The mean burst spectrum in our energy range can be represented as:

$$
\left.<N_{E}(E)\right\rangle=[0.91 \pm 1.9](E / \mathrm{MeV})^{-[2.43 \pm 0.56]} \text { photons } \mathrm{cm}^{-2} \mathrm{~s}^{-1} \mathrm{MeV}^{-1} .
$$

Here the uncertainties are from the square root of the sample variance, summed in quadrature with the mean statistical error, and so indicate the wide variety of burst spectra measured by COMPTEL. This power-law shape $>1 \mathrm{MeV}$ is consistent both 


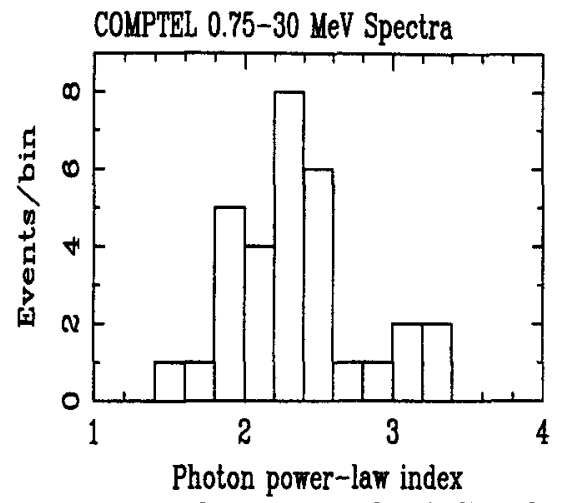

FIGURE 2. Histogram of photon power-law indices for COMPTEL bursts.

with earlier results from SMM [10] and higher energy measurements from CGROEGRET, when they are available [11-13]. For a few bright bursts, COMPTEL has measured curvature in its energy range, consistent with a peak in $\nu F_{\nu}$ of $\sim 1 \mathrm{MeV}$ rather than hundreds of $\mathrm{keV}$ [6-9]. No significant line features have been detected in any individual burst. This, together with lower energy OSSE and BATSE results, [14-16], shows that gamma-ray burst spectra seem to have a canonical shape: a peak in $\nu F_{\nu}$ space typically centered around $\sim$ hundreds of $\mathrm{keV}$ (but with a range of about a factor of $\mathbf{5}$ in either direction); at higher energies, a power-law tail extending out as far as it is measureable, sometimes to $100 \mathrm{MeV}$ or more; and indications of flattening or turnovers at X-ray energies [17].

Motivated by findings at lower energies of two classes of burst emission based on hardness [20,21] we tested the spectral index histogram for evidence of two populations: one with $(\langle\alpha\rangle=2.2)$; and one softer $\left(\left\langle\alpha_{2}\right\rangle=3.2\right)$. However the drop in $2 \times \log$-likelihood was only 7.5 for an addition of 3 degrees of freedom, so this was not significant.

The dramatic detections of optical counterparts for two recent bright bursts, one associated with a galaxy [18] and the other consistent with a redshift $z \sim 0.83$ [19], have focused attention on cosmological "fireball" models, and in particular on relativistic shocks. In these scenarios, the canonical break at several hundred $\mathrm{keV}$ is attributed to either inverse Compton, or synchrotron processes [22-24]. However others emphasize the difficulties of realistically producing gamma-rays with complex time-histories this way, one alternative being pulsar-like emission from the debris of a collapsed neutron star $[25,26]$. As these various models mature, the variability, break energy and power-law high energy emission remain strong constraints on the physics of realistic emission mechanisms. 

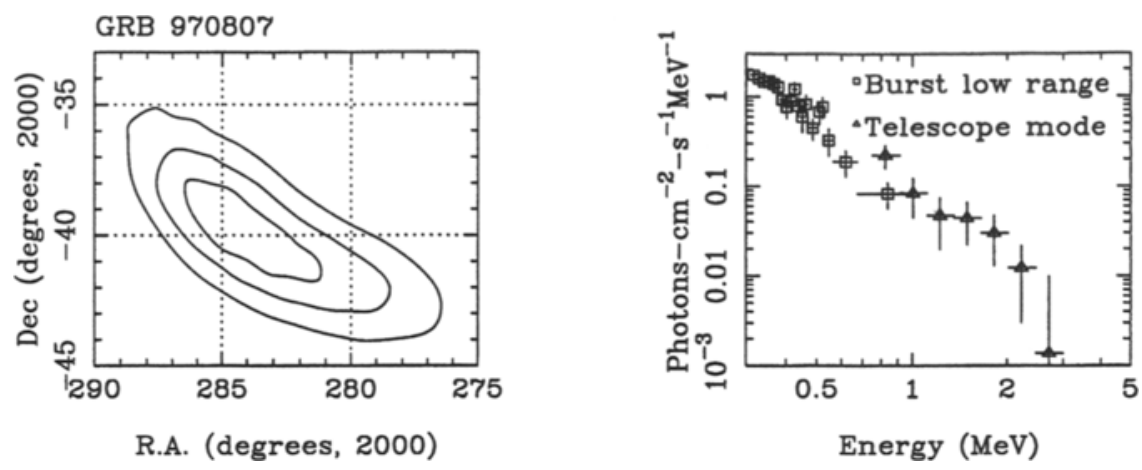

FIGURE 3. COMPTEL 1,2 , and $3 \sigma$ position contours (left) and low-range burst detector plus telescope spectra (right) for GRB 970807.

\section{RAPID BURST RESPONSE}

In a joint project with BACODINE/GCN, COMPTEL automatically processes any burst (defined by a BATSE burst trigger) within $65^{\circ}$ of telescope zenith. If the burst is localized, an initial $\sim 1-2^{\circ}$ position can be sent to a world-wide network of ground-based observers within $\sim 7.5$ minutes of burst onset. These error boxes are considerably larger than those available from BeppoSAX or RXTE, but the response time is minutes rather than hours. Our ground-based observers include several with large field of view Schmidt cameras, capable of rapid response [27]. Out of, on average, $\sim 70$ BATSE triggers per year in our field of view, we successfully localize $\sim 5$. We are now upgrading the automated burst response, both to at least halve the response time, and to make the system more robust.

\section{THE MOST RECENT COMPTEL TELESCOPE BURST}

The most recent burst (GRB 970807) was quite soft, so intervention by the duty scientists was necessary. This position did not get broadcast until $\sim 45$ minutes after burst onset. On the left in Figure 3, we display the (statistical) burst location contours. The COMPTEL $2 \sigma$ contours overlap the BATSE and IPN positions. On the right are the best-fit telescope mode plus low-range burst-mode spectra, assuming a $48 \mathrm{~s}$ integration time. The best fit to the burstmode was $N_{E}(E) \sim(0.05 \pm 0.01) E^{-3.2 \pm 0.2}$, while for the telescope data it was $N_{E}(E) \sim(0.1 \pm 0.03) E^{-3.3 \pm 1.1}$ Although it is soft, it is consistent with the range of spectra previously measured by COMPTEL. 


\section{CONCLUSION}

COMPTEL continues to measure MeV spectra and locations of bursts in its field of view, at an average rate of $\sim 5$ per year. We are in the process of upgrading our rapid response, bringing with it the possibility, for particularly well-suited bursts, of broadcasting $\sim 1-2^{\circ}$ localizations while the intense portion of the burst is still underway.

The COMPTEL project is supported in part through NASA grant NAS 5-26646, DARA grant 50 QV 90968, and the Netherlands Organization for Scientific Research (NWO).

\section{REFERENCES}

1. Schönfelder, V., et al., ApJS 86, 629 (1993).

2. Kippen, R. M., et al, $A p J$, in press (1998a).

3. Kippen, R. M., et al, Adv. Space Res., in press (1998b).

4. Kippen, R. M., et al., Gamma-Ray Bursts, ed C. Kouveliotou, M. S. Briggs \& G. J. Fishman, (New York: AIP) 384197 (1996).

5. Connors, A., et al., in Proc. of the 25th ICRC (1997).

6. Winkler, C., et al., Ap\&SS 231, 153 (1995a).

7. Winkler, C., et al., $A \mathscr{E} A$ 302, 765 (1995b).

8. Hanlon, L. O., et al., Ap\&SS 231, 157 (1995).

9. Hanlon, L. O., et al., $A \& A$ 285, 161 (1994).

10. Matz, S. M., et al., ApJ 288, L37 (1985).

11. Dingus, B., et al., Ap\&SS 231, 187 (1995)

12. Catelli, J., et al., Gamma-Ray Bursts, ed. C. Kouveliotou, M. S. Briggs \& G. J. Fishman, (New York: AIP) 384, 158 (1996).

13. Hurley et al., Nature 372,652 (1994).

14. Share, G. H., et al., Gamma-Ray Bursts, ed. G. J. Fishman, J. J. Brainerd \& K. Hurley, (New York: AIP) 307, p. 283 (1994).

15. Schaefer, B. E., et al., ApJ, in press (1998).

16. Band, D. L., et al., ApJ 413, 281 (1993).

17. Strohmayer, T., et al., ApJ, submitted (1998).

18. van Paradijs, J., et al., Nature 386, 686 (1997).

19. Metzger, M. R., et al., Nature 387, 878 (1997).

20. Pendleton, G. N., et al., $A p J 489,175$, (1997).

21. Belli, M., ApJ 479, L31 (1997).

22. Mészáros, P., and Rees, M. J., ApJ 476, 232 (1997).

23. Pilla, R., and Loeb, A., in Proc. of the VIII Marcel Grossman Meeting on General Relativity, eds. R. Ruffini and T. Piran (World Scientific, Singapore) (1997).

24. Cohen, E., et al., ApJ 488, 330 (1997).

25. Katz, J., $A p J$, submitted (1997).

26. Sari, R., and Piran, T., ApJ, submitted (astro-ph/9701002) (1997).

27. McNamara, B. J., et al., ApJS 103, 173 (1996). 